\title{
Zur Theorie der Muskelkontraktion.
}

Kann die Muskelkraft durch osmotischen Druck oder Quellungsdruck erzeugt werden?

Von

J. Berustein.

(Mit 1 Textfigur.)

I.

Mancherlei Bewegungsvorgänge und Formveränderungen bei den Pflanzen beruhen, wie man annimmt, zum grossen Teile auf osmotischen Prozessen. Der Turgor der Pflanzenzelle wird nach Pfeffer auf den osmotischen Druck ihres Inhaltes zurückgeführt. Die Aufsaugung des Wassers in der Pflanze muss daher vornehmlich auf Osmose beruhen, und die durch den Turgor bewirkte Aufrichtung der Pflanzenteile ist also eine durch osmotischen Druck hervorgebrachte Bewegung. Unzweifelhaft ist auch der osmotische Druck der tierischen Zelle ein wichtiger Faktor bei der Erhaltung ihres Turgors, da man ihren Protoplasten, ebenso wie den der Pflanzenzellen, als eine für viele Stoffe semipermeable Membran erkannt hat.

Aus diesen Gründen liegt es nahe, sich die Frage vorzulegen, ob die Kontraktionskraft des Muskels sich vielleicht auf einen bei der Reizung desselben entstehenden stärkeren osmotischen Druck in gewissen Elementen der Muskelfaser zurückführen lasse. Die Entscheidung derselben ist deshalb von Bedeutung, weil, wenn sich ein negatives Resultat ergibt, andere zur Erklärung der Kontraktion aufgestelite Theorien an Wahrscheinlichkeit dadurch gewinnen würden. Ich habe bei der Veröffentlichung der von mir aufgestellten Oberflächenspannungstheorie ${ }^{1}$ ) der Kontraktion eine solche Möglichkeit nicht erwähnt, weil sie bis dahin meines Wissens von niemandem ausgesprochen war. Indessen war eine Vorstellung

1) Die Energie des Muskels als Oberflächenenergie. Dieses Archiv Bd. 85 S. 271. 1901.

E. Pflüger, Archir für Physiologie. Bd. 109. 
ähnlicher Art gelegentlich von Reuleaux ${ }^{1}$ ) in sinnreicher Form gegeben worden. Er verglich die Muskelfaser mit einem mit Flüssigkeit gefüllten Schlauch, der auch in mehrere blasenförmige Querstücke zerfallen kann, und dachte sich, dass durch einen inneren Prozess bei der Reizung Flüssigkeit in diese Blasen eingetrieben werde. Es würden sich dann die durch ein Gewicht gedehnten länglichen Blasen zu Kugeln abzurunden suchen und der ganze Schlauch würde sich verkürzen. Ein Modell dieser Art hat Reuleaux angegeben und abgebildet.

Eine ähnliche Vorstellung ist von W. M'Donga $1^{2}$ ) ausgesprochen worden. Er denkt sich nach Bütschli die Substanz der Fibrillen mit kleinen wabenartigen Hohlräumen erfült, welche Flüssigkeit enthalten und sich bei der Kontraktion durch Wachsen des osmotischen Druckes stärker mit Flüssigkeit anfüllen, welche aus dem Sarkoplasma resp. der Parenchymflüssigkeit hineindiffundiert. Haben diese Hohlräume, besonders im gedehnten Muskel, eine längliche Form, so würden sie sich bei der Reizung. $\mathrm{zu}$ einer Kugel abzurunden suchen und sich unter Zunahme ihrer Dicke verkürzen. Die von Reuleaux angenommene Kraft würde also in diesen Fällen osmotischer Druck sein und die von Reuleaux ersonnene Mechanik könnte in Aktion treten.

Diese Theorie erfüllt zwar die bekannte Forderung, dass das Volumen des Muskels bei der Kontraktion konstant bleibt, aber man kann schon gegen ihre Mechanik den Einwand erheben, dass die Verkürzung der Hohlräume durch ein Maximum in Verlängerung übergehen müsse, wenn die eintretende Flüssigkeitsmenge weiter zunimmt. Letztere hat freilich eine Grenze in dem im Gewebe vorhandenen Vorrat. $\mathrm{Zu}$ ihrer Stütze könnte man sogar die Engelmann'sche Beobachtung anführen, dass die anisotropen Scheiben auf Kosten der isotropen der Fibrille an Volumen bei der Verkürzung zunehmen, unter der Annahme, dass dieser Vorgang auf Osmose beruhe.

In der Tat muss durch den chemischen Prozess bei der Kontraktion der osmotische Druck sich erhöhen. Es entstehen durch

1) Die praktischen Beziehungen der Kinematik zu Geometrie und Mechanik S. 772 u. ff, Braunschweig 1900.

2) A theory of muscular contraction. Journ. of dnatom. and Physiol. vol, 32 p. 187. 1898 . 
Spaltung und Oxydation aus komplizierten organischen Molekülen eine grössere Zahl einfacher Moleküle, so dass die molekulare Konzentration und damit der osmotische Druck im Muskel dementsprechend ansteigt. Bei der Erschlaffung kann durch Diffusion eine Abgabe der entstandenen Moleküle aus den kontraktilen Elementen der Faser an die indifferenten Elemente stattfinden. Aus der Muskelchemie wissen wir, dass nach übereinstimmenden Untersuchungen von Voit und neueren von Zuntz die $\mathrm{N}$-losen Nälirstoffe zur Erzeugung der Muskelarbeit unter normalen Bedingungen (bei Ausschluss von Überanstrengungen) vollständig ausreichen, und dass dabei ein Mehrverbrauch von Eiweiss gegenüber der̃ Muskelruhe nicht stattfindet. Als direktes Brennmaterial der Muskelmaschine sind bis jetzt nur die Kohlehydrate nachgewiesen, also das Glykogen resp. der daraus gebildete Zucker. Wenn auch von Pflüger gezeigt worden ist, dass bei fast ausschliesslicher Eiweissnahrung (mageres Fleisch) erhebliche Muskelleistungen stattfinden können, so ist doch zu vermuten, dass unter dieser Bedingung die Leber aus dem Eiweiss das zur Unterhaltung der Muskelarbeit nötige Glykogen bereitet. Ähnlich dürfte es sich mit dem Fett der Nahrung bei Ausschluss von Kohlehydraten verhalten. Doch möge dem sein wie ihm wolle, wir werden erst eine Berechnung aufstellen unter der wahrscheinlichsten Annahme, dass der Zucker (Dextrose) das Brennmaterial des Muskels bildet, und dann diese Berechnung auch auf das Fett und das Eiweiss ausdehnen.

Das Prinzip der Berechnung ist folgendes. Wir denken uns einen Kubikzentimeter Muskel aus parallelen Fasern von $1 \mathrm{~cm}$ Länge und $1 \mathrm{qcm}$ Querschnitt. Bei einer isometrischen Zuckung (Muskel festgespannt), deren Kraft gemessen wird, mögen $n g$ Moleküle Zucker verbrannt werden. Wir wollen annehmen, dass die Verbrennung von $\mathrm{C}_{6} \mathrm{H}_{12} \mathrm{O}_{6}$ eine vollständige $\mathrm{zu} 6 \mathrm{CO}_{2}+6 \mathrm{H}_{2} \mathrm{O}$ sei, und dass keine teilweise Spaltung in Milchsäure $\left(\mathrm{C}_{3} \mathrm{H}_{6} \mathrm{O}_{3}\right)$ eintrete, wobei der osmotische Druck kleiner ausfallen würde. Es vermehrt sich daher die Zahl der Moleküle im Verhältnis von $1: 12$. Von diesen müssen wir aber die Wassermoleküle abrechnen, da sie den osmotischen Druck nicht erhöhen, sondern im Gegenteil vermindern, was wir aber getrost vernachlässigen können in Anbetracht der sehr kleinen dabei entstehenden Wassermenge. Unter dieser Voraussetzung vermehrt sich die Zahl der Moleküle nur im Verhältnis von 1:6, und die Steigerung des osmotischen Druckes bei der Kontraktion entsteht 
demnach durch Bildung von $6 n \mathrm{~g}$ Mol. an Stelle von $n$ g Molo oder

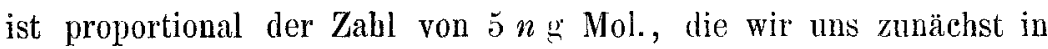
1 cem verteilt denken wollen. Man könnte aber auch zweitens annehmen, dass $6 \mathrm{CO}_{3} \mathrm{H}_{2}$ entstehen, welche in die Ionen $6 \mathrm{CO}_{3}+12 \mathrm{H}$ dissoziieren. Für diesen Fall würde für die Zahl 5 die Zahl 18 --1 =-17 zu setzen sein, und die Steigerung des osmotischen Druckes bei der Kontraktion würde von $17 n \mathrm{~g}$ Mol. (Ionen) abhängen. Der Wert dieses osmotischen Druckes in $1 \mathrm{ccm}$ Muskel lässt sich nach bekannten Daten der physikalischen Chemie leicht berechmen.

Nach A. Fick (Gesammelte Abhandlungen S. 131) erzeugt $1 \mathrm{~g}$ Froschmuskel bei einer energischen Znckung gegen grossen Widerstand (isometrisch) eine Wärmemenge von $3 \mathrm{mg}$ Kal. Nimmt man die Verbrennungswärme des Zuckers zu $3277 \mathrm{~g}$ Kal. auf $1 \mathrm{~g}$ an, so entstehen $3 \mathrm{mg}$ Kal. aus $\frac{3}{3277 \cdot 10^{8}}$ g Zucker. Das spezifische Gewicht des Muskels zu 1,06 angenommen und das Mol. des Zuckers $=180 \mathrm{~g}$ gesetzt, so werden in $1 \mathrm{ccm}$ Muskel $n=\frac{3 \cdot 1,06}{180 \cdot 3277 \cdot 10^{3}} \stackrel{\text { Mol. }}{ }$ Zucker verbraucht.

$1 \mathrm{~g} \mathrm{Mol}$. in $1 \mathrm{ccm}$ Flüssigkeit gelöst erzengt bekanntlich einen osmotischen Druck ${ }^{1}$ ) von $49,3 \cdot 34200 \cdot 13,59 \mathrm{~g}$ auf $1 \mathrm{qcm}$.

Wenn nun in einem Muskel aus $n \mathrm{~g}$ Mol. Zucker $5 n \mathrm{~g} \mathrm{Mol}$. entstehen, so ist der dadurch erzeugte osmotische Druck

$$
p=\frac{5 \cdot 3 \cdot 1,06 \cdot 49,3 \cdot 34200 \cdot 13,59}{180 \cdot 3277 \cdot 10^{8}}=0,62 \mathrm{~g} \text {. }
$$

Oder wenn aus $n \mathrm{~g}$ Mol. Zucker $17 n g$ Mol. entstehen, so ist $p=2,14 \mathrm{~g}$.

Vergleicht man mit diesem Druckwerte die Muskelkraft für 1 qem Muskel (absolute Muskelkraft), so ergibt sich, dass erstere gegen letztere ausserordentlich gering ist. Die absolute Muskelkraft im Tetanus kann man für den Froschmuskel zu etwa $3000 \mathrm{~g}$ annehmen, die Kraft einer isometrischen Zuekung daher mindestens zu $600 \mathrm{~g}$. Es fragt sich daher, ob irgendeine Mechanik im Muskel denkbar ist, vermöge deren so kleine osmotische Druckwerte so sehr viel grössere mechanische Spannungen zu erzeugen imstande sind.

1) $49,3 \mathrm{~cm} \mathrm{Hg}$ Druck ist der osmotische Druck einer $1 \%$ igen Rohrzuckerlösung bei $18^{\circ}$ C., 342 ist das Molekulargewicht des Rohrzuckers und 13,59 das spez. Gewicht des $\mathrm{Hg}$. 
Führen wir noch dieselbe Berechnung unter der Annahme aus, dass statt des Zuckers bei der Kontraktion Fett oder Eiweiss verbrannt wird, so ergeben sich folgende Resultate.

Für Fett erhalten wir den Wert

$$
\left.p=\frac{171 \cdot 3 \cdot 1,06 \cdot 49,3 \cdot 34200 \cdot 13,59}{890 \cdot 9069 \cdot 10^{3}}=1,57 \mathrm{~g}^{1}\right) .
$$

Für Eiweiss erhalten wir nach der von $\mathrm{H}_{0}$ fmeister ${ }^{2}$ ) angegebenen chemischen Formel:

$$
p=\frac{1484 \cdot 3 \cdot 1,06 \cdot 49,3 \cdot 34200 \cdot 13,59}{10166 \cdot 4998 \cdot 10^{3}}=2,13 \mathrm{~g} .
$$

Also auch für diesen Fall ist der osmotische Druck sehr.viel kleiner als die entsprechende Muskelkraft, für das Fett als Kraftquelle kleiner, für das Eiweiss etwa ebenso gross als für den Zucker.

Man müsste nun allerdings diesen Wert des osmotischen Druckes noch etwas vergrössern, wenn man berücksichtigt, dass die kontraktilen Elemente nur einen Teil der ganzen Muskelmasse bilden, und dass demnach nur in diesem Teil der osmotische Druck wachsen würde, während der übrige Teil das Wasser hergeben würde, welches diesem Drucke folgt. Nun muss man aber unbedingt mindestens die gesamte doppelbrechende Substanz als die kontraktile ansehen, und da diese ungefähr $1 / 3-1 / 2$ der ganzen Muskelmasse ausfüllt, so würden wir, wenn wir den Raum eines Kubikzentimeter Muskel auf $1 / 3-1 / 2$ beschränken, in diesem doch nur den doppelten bis dreifachen der gefundenen Werte für den osmotischen Druck bei der Kontraktion erhalten, - Werte, welche immer noch weit unterhalb der Muskelkraft liegen.

\section{II.}

Nach Anstellung dieser Rechnungen haben wir nun zu überlegen, ob irgendeine in der Muskelfaser mögliche Mechanik denkbar ist, durch welcbe so kleine osmotische Kräfte sich $z u$ einer viel grösseren Kraft summmieren könnten. Nebmen wir die osmotische Kraft in maximo zu

1) Molekulargewicht des Stearin $\left(\mathrm{C}_{57} \mathrm{H}_{110} \mathrm{O}_{6}\right)=890$, Verbrennungswärme desselben $=9069$, Zahl der aus $1 \mathrm{Mol}$. entstehenden Moleküle $\mathrm{CO}_{2}=57$ und Ionen $\mathrm{CO}_{3}, 2 \mathrm{H}=171$.

2) Siehe Cohpheim, Chemie der Eiweisskörper S. 18.

Molekulargewicht des Eiweisses $\left(\mathrm{C}_{450} \mathrm{H}_{720} \mathrm{~N}_{116} \mathrm{~S}_{6} \mathrm{O}_{140}\right)=10166$. Verbrennungswärme $=4998$. Zahl der entstehenden Moleküle und Ionen, wenn aller $\mathrm{C} z u$ $\mathrm{CO}_{2}$ und aller $\mathrm{N}$ zu $\mathrm{NH}_{3}$ verwendet wird, $=1484$. 
$3 \times 2,14$, rund zu $6 \mathrm{~g}$ und die Muskelkraft rund $\mathrm{zu}$ b00 $\mathrm{g}$ an, so müsste dabei eine Steigerung der Kraft un das Hundertfache eintreten.

Zuerst wollen wir den Fall in Betracht ziehen, dass in der kontraktilen Substanz sich kleine länglicbe, mit flüssiger (resp. zäbflüssiger) Masse gefüllte Hohlräume befinden, welche von elastischen Wänden begrenzt sind, und in der Querrichtung der Faser so nebeneinander gelagert sind, dass ihre Längsrichtung mit der der Faser parallel ist. Das wäre diejenige Struktur und Annahme, welche M'Dougall seiner Theorie zugrunde zu legen scheint. In der Tat ist dies eine Annahme, welche anscheinend mit den mikroskopischen Beobachtungen über die Struktur der Muskelfasern nicht im Widerspruch steht. M'Dougall betrachtet die Segmente der Fibrille, welche er Sarkomere nennt, als solche Gebilde. Wenn wir aber voraussetzen, dass diese schlauchförmigen Gebilde von elastischen Wänden eingeschlossen sind, und dass in ihnen bei der Kontraktion der innere Druck durch Osmose steigt, so ist leicht einzusehen, dass dieselben unter dieser Bedingung nicht imstande sein würden, sich zu verkürzen. Haben wir irgend einen mit Flüssigkeit gefüllten elastischen Schlauch und erhöhen in denselben durch Verbindung mit einem Druckreservoir den inneren Druck, so dehnt sich derselbe nicht nur in der Querrichtung, sondern auch in der Längsrichtung aus und verlängert sich daher, anstatt sich zu verkürzen. Der Versuch lässt sich leicht mit einem gewöhnlichen Gummischlauch von mässiger Dicke und beliebiger Länge ausfübren, wenn man ihn mit einem Glasrohr oben verbindet, an demselben senkrecht aufhängt, unten zubindet und mit einem mässigen Gewicht belastet. Ist der Schlauch mit Wasser oder auch mit Luft gefüllt, und setzt man seinen Inhalt einem angemessenen Druck durch Verbindung des Glasrobres mit der Wasserleitung aus, so sieht man deutlich, dass er sich gleichzeitig verdickt und verlängert; das Gewicht sinkt ${ }^{1}$ ).

Es ist klar, dass für den Muskel ausser dem Zylinder noch andere aber nur Rotationskörper in Frage kommen können, denn im anderen Falle könnte die Verdickung des Muskels nicht nach allen Richtungen des Quersehnittes eine gleiche sein. Was nun in dieser Hinsicht von einem zylindrischen Rohr gilt, das wird mit grosser Wahrscheinlichkeit auch für jeden von konvexen Fläehen begrenzten

1) Für ein zylindrisches Rohr ist dieses Verhalten streng bewiesen in dem Werke von C. Bach, Elastizität und Festigkeit. 4. Aufl, S. 528 u. ff., siehe S. 536 , Anmerk. 1. Berlin 1902. 
Rotationskörper mit elastisch gespannten Wandungen gelten. Für ein Rotationselipsoid würde sich dies wobl am leichtesten theoretisch beweisen lassen. Ich beschränke mich aber darauf, dies an einem eliptisch gestalteten Gummiballon experimentell nachzuweisen. Ist derselbe vollständig aufgeblasen, mit irgendeinem Gewichte belastet, so tritt bei Verstärkung des Druckes Verlängerung desselben ein, wie unten angeführte Experimente zeigen.

Es fragt sich nun, ob anders gestaltete, mit Flüssigkeit gefüllte Hohlkörper eine Verkürzung ergeben können und unter welchen $\mathrm{Be}$ dingungen dies geschieht. Ein solcher Fall ist in der Tat in dem oben erwähnten Reuleaux'schen Modell verwirklicht. Reuleaux hat indes die zum Gelingen des Versuchs erforderlichen Bedingungen nicht genauer angegeben. Wir wollen daher zuerst einen Versuch beschreiben, bei welchem man eine Verkürzung einer Reuleaux'schen Blase am deutlichsten feststellen kann. Man nehme einen im aufgeblasenen Zustande etwa kugelförmigen Ballon. Am besten würden wir uns denselben aus einem Luftballonstoff (gefirnisste Seide) herstellen, indessen kann man dazu auch einen Gummiballon oder eine feuchte Harnblase anwenden. Der Ballon wird an seinem offenen Ende mit einem Rohr verbunden, an demselben in zusammengefaltetem leeren Zustande senkrecht aufgehängt und an seinem unteren geschlossenen Ende mit einer leichten Wagschale versehen, auf die wir ein angemessenes Gewicht legen. Die Länge des leeren Ballons ist dann nahezu gleich der halben Peripherie der aufgeblasenen Ballonkugel. Blasen wir nun den Ballon auf - der Versuch ist bequemer mit Luft als mit Wasser auszuführen - so verkürzt er sich, indem er sich der Kugel zu nähern strebt und hebt dabei das Gewicht.

Ich habe diesen Versuch mit einem Gummiballon ausgeführt, welcher etwa $5 \mathrm{~cm}$ Länge im zusammengefalteten Zustande ohne oder mit geringer Belastung hatte. Das zuführende Rohr wurde mit einem Druckballon verbunden, der durch eine Presse mit Schraube komprimiert werden konnte; ein Seitenrohr führte zu einem Quecksilbermanometer, an welchem man den herrschenden Druck ablesen konnte. Es wurde auf diese Weise der Druck gemessen, welcher zur Hebung verschiedener Gewichte erforderlich war, und die Höhe, auf welche sie gehoben wurden. Es konnte auch der Druck ermittelt werden, welcher zum Abheben verschiedener Gewichte von einer Unterlage eben hinreichte, ein Versuch, welcher der Kraftmessung: am Muskel entsprechen würde. Die angehängte leichte Wagschale 
aus Aluminium wurde als Belastung Null angenommen, die Hubhöhe wurde mit Hilfe eines dünnen Rohrstäbchens, welches am unteren Ende des Ballon horizontal befestigt war und vor einer Skala spielte, gemessen. Es wurde ferner die jedesmalige Debnung durch die Gewichte von $5 \mathrm{~cm}$ ursprünglicher Länge aus abgelesen ${ }^{1}$ ). Der Ballon wurde beim Aufblasen im Querschnitt keineswegs kreisrund, und daher wurden der grösste und kleinste Durchmesser desselben in aufgeblasenem Zustande bei jeder Belastung gemessen.

Versuch 1.

\begin{tabular}{r|c|c|c|c|c|c}
\hline Nr. & $\begin{array}{c}\text { Belastung } \\
\mathrm{g}\end{array}$ & $\begin{array}{c}\text { Dehnung } \\
\mathrm{mm}\end{array}$ & $\begin{array}{c}\text { Hubhöhe } \\
\mathrm{mm}\end{array}$ & $\begin{array}{c}\text { Arbeit } \\
\mathrm{gmm}\end{array}$ & $\begin{array}{c}\text { Druck } \\
\mathrm{mm} \mathrm{Hg}\end{array}$ & $\begin{array}{c}\text { Durehmesser des } \\
\text { Hallon } \\
\text { mm }\end{array}$ \\
\hline 1 & 0 & 0 & 7,5 & 0 & 12,5 & $37-28$ \\
2 & 100 & 3 & 6,0 & 600 & 15,0 & $37-27$ \\
3 & 200 & 6 & 4,0 & 800 & 17,5 & $35-25$ \\
4 & 300 & 10,5 & 3,25 & $\mathbf{6 7 5}$ & 17,5 & $34-24$ \\
5 & 400 & 15,0 & 2,0 & 800 & 17,5 & $33-23$ \\
6 & 500 & 20,5 & $\mathbf{1 , 5}$ & 750 & 19 & $32-22$ \\
7 & 400 & 17,0 & 2,3 & 920 & 17,5 & $33-23$ \\
8 & 300 & 13,7 & 3,9 & $\mathbf{1 1 7 0}$ & 17,5 & $34-24$ \\
9 & 200 & 9,5 & 5,2 & 1040 & $?$ & $35-25$ \\
10 & 100 & 5,2 & 6,2 & 620 & 18 & $37-27$ \\
11 & 0 & 3,0 & 8,0 & 0 & 15 & $37-27$
\end{tabular}

In dem angeführten Versuch zeigte der Ballon manche auffallende Ähnlichkeit in seinem Verhalten mit dem Muskel. Man sieht erstens, dass die Hubhöhe mit zunehmender Belastung abnimmt und zweitens, dass sogar die Arbeit mit zunehmender Belastung ein Maximum erreicht und bei weiterer Zunahme derselben wieder abnimmt. Wir sehen beim Hin- und Rückgang der Belastungen bis $300 \mathrm{~g}$ ein Maximum der Arbeit von 975 und $1170 \mathrm{gmm}$ eintreten. Beim Rückgang sind die Dehnungen infolge der elastischen Nachwirkung grösser und daher auch die Hübe und Arbeiten. Die Drucke wachsen mit zunehmender Belastung erst schneller, dann langsamer und erscheinen beim Rückgang auch grösser als beim Hingang.

Trotz dieser Ähnlichkeiten mit dem Muskel, die zu einer Theorie der Kontraktion verlocken könnten, müssen wir von vornherein eine darauf gegründete als unwabrscheinlich erklären. Denn erstens gibt es in dem mikroskopischen Bilde der Muskelfaser wohl keinen Anhalt für das Vorhandensein von kleinen Hohlräumen init längsgefalteten

1) Bei weiteren Versuchen dieser Art würde es sich lohnen, die Hubhöhen myographisch zu verzeichnen. 
Wandungen, welche sich durch Anfüllung aufblähen. Zweitens müsste mit zunehmender Füllung derselben nach dem Maximum der Verkürzung eine Verlängerung des Muskels erfolgen, und zwar um so eher bei weiterer Reizverstärkung, je grösser das angehängte Gewicht ist. In dem angeführten Versuche erfolgte jedesmal, wenn der Druck die bei jeder Belastung angegebene Grösse überstieg, eine Senkung des Zeigers an der Skala. Drittens müssten in der Muskelfaser resp. den Fibrillen derselben ausser den kontraktilen Hoblräumen noch disponible Volumina vorhanden sein, in denen sich der erforderliche Wasservorrat in der Ruhe befindet, der bei der Kontraktion in die kontraktilen Hohlräume diffundiert. Diese „Wasserräume" könnten nicht ausschliesslich in demselben Querschnittselement der Faser zwischen den kontraktilen Räumen liegen, weil sonst eine der Verkürzung entsprechende Querschnittszunahme kaum stattfinden könnte, wie es in Wirklichkeit der Fall ist. Dagegen könnte man sich mit der Annabme helfen, dass bei der quergestreiften Faser die kontraktilen Hohlräume nur in den doppelbrechenden, die Wasserräume in den einfach brechenden Scheiben liegen - was, wie schon gesagt, an die Engelmann'sche Folgerung aus dem mikroskopischen Bilde der beiden Substanzen der Fibrille bei der Kontraktion erinnert.

Von wesentlicher Bedeutung ist indes die Vergleichung der Muskelkraft mit der in dem Modellversuch beobachteten Kraftgrösse. Die Kraft der Verkürzung können wir an dem Modell nach der Weber'schen Belastungsmethode und nach der Rosentha l'schen Überlastungsmethode messen. In dem Versuche wurden $100 \mathrm{~g}$ um $3 \mathrm{~mm}$ über die natürliche Länge gehoben, $200 \mathrm{~g}$ blieben $2 \mathrm{~mm}$ unter der natürlichen Länge zurück. Der Kraftwert bei natürlicher Länge wđ̆rde etwa bei $160 \mathrm{~g}$ liegen. Diese Kraft bätte man zu beziehen auf den grössten Querschnitt, den der Ballon beim Aufblasen unter dieser Spannung annimmt. Dieser lässt sich aus den angegebenen Durchmessern des Ballons nur annähernd berechnen ${ }^{1}$ ), und man würde auf diese Weise für die auf $1 \mathrm{qcm}$ reduzierte Kraft etwa $21,29 \mathrm{~g}$ erhalten. Nun kann man ferner aus dem am Manometer abgelesenen Druck die auf $1 \mathrm{qcm}$ der Wandung wirkende Druckkraft berechnen. Nimmt man für $160 \mathrm{~g}$ Belastung durch Interpolation einen Druck von $16,9 \mathrm{~mm} \mathrm{Hg}$. an, so erhält man für $1 \mathrm{qcm}$ eine Druckkraft von 1,69·13,5 $=22,8 \mathrm{~g}$.

1) Aus den beiden Durchmessern wurde ein mittlerer zu $31 \mathrm{~mm}$ angenommen und daraus die Fläche als kreisförmige berechnet. 
Man sieht also, dass unter den gegehenen Verhältnissen des angestellten Versuches die Verkürzungskraft des Ballons auf $1 \mathrm{qcm}$ (absolute Kraft) dem innern Druck auf diese Fläche ungefähr gleich kam. In einigen anderen Versuchen dieser Art erhielt ich ganz ähnliche Werte und ebenso auch bei dem Überlastungsverfahren.

Wenn nun in der Muskelfaser bei einer solchen Einrichtung die Verhältnisse ähnliche sein würden wie in dem benutzten Kautschukballon, so wäre es damit experimentell bewiesen, dass diese Kraft ungefähr nur so gross sein könnte, als der in den gedachten Hohlräumen herrschende osmotische Druck, und es fragt sich nun, $o b$ dieser bei dem geringen Werte von höchstens $6 \mathrm{~g}$ ausreichen könnte, uin die Muskelkraft zu erzengen.

Hierbei müssen wir nun in Betracht ziehen, dass wir den Muskel keineswegs mit einem einzelnen Ballon vergleichen dürfen, vielmehr hätten wir uns vorzustellen, dass auf einem Quadratzentimeter Querschnitt eine grosse Zahl von kleinen Ballons nebeneinander gereiht wären, von denen jeder einzelne dieselben Verhältnisse in seinen Dimensionen und elastischen Konstanten haben möge wie der untersuchte Ballon. Hierzu kommt für den Muskel noch die Forderung,
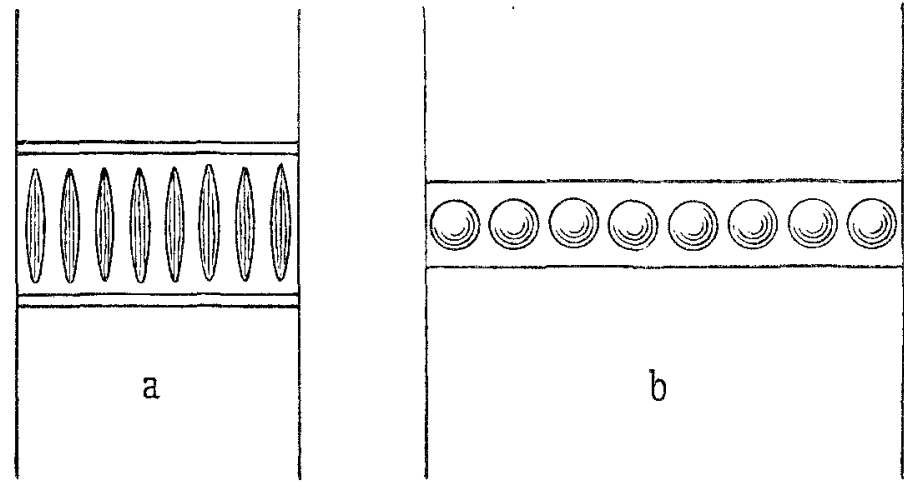

Fig. 1.

dass das Volumen des ganzen Systems, d. h. der Ballons plus der Masse, in welcher sie eingebettet sind, bei der Verkürzung konstant bleibt, während an dem untersuchten Ballon das Volumen bei der Aufblähung und Verkürzung augenscheinlich erheblich zunimmt, da der Inhalt von aussen her hineingepresst wird. In dem Muskel aber und in jeder Faser muss die in die kleinen Ballons eintretende Flussigkeitsmasse schon vorher enthalten gewesen sein. Um sich 
eine Vorstellung von der Sache zu machen, sei in Fig. 1 a ein Segment der ruhenden, in Fig. $1 b$ ein Segment der kontrahierten Muskelfaser dargestellt, deren Gesanitvolumina konstant seien. Die Volumina der kleinen Ballons müssen also bei der Kontraktion um ebensoviel zunehmen, als das Volumen der Zwischen- und Nebenschichten abnimmt.

Eine allgemeine analytische Behandlung des Problems bietet erhebliche Schwierigkeiten dar und erscheint mir daher wenig lobnend.

Folgende Betrachtung lässt indes eine ungefähre Berechnung $\mathrm{zu}$, wenn man $\mathrm{zu}$ einer Kraft von $600 \mathrm{~g}$ auf $1 \mathrm{qcm}$ Muskel gelangen will. Man denke sich einen Ballon, der im gefüllten Zustande einen maximalen Quersehnitt von $1 \mathrm{qcm}$ hat und die Kraft des berechneten osmotischen Druckes von $6 \mathrm{~g}$ besitzt. Die Länge desselben sei $L$, der mittlere Querschnitt seiner Wandung (nicht zu verwechseln mit dem des Volumens) in der ganzen Peripherie sei $Q^{1}$ ). Die Dehnung desselben im zusammengefalteten Zustande durch das Gewicht $P=6 \mathrm{~g}$ sei $D$. Nun denken wir an Stelle dieses Ballons $n$ kleine Ballons in einem Quadratzentimeter Querschnitt des Systems nebeneinander gereiht (wie in Fig. 1), deren Wandungen dieselbe Beschaffenheit und Dicke haben mögen wie jener; die Länge eines solchen sei $l$, der mittlere Quersehnitt der Wandungen $q$, und das Gewicht $p$, welches der Kraft dieses gleich ist, dehne denselben um $d$. Für diesen Fall können wir annähernd setzen:

$$
\begin{gathered}
L: D=l: d, \\
P=\frac{D \cdot Q}{L} \cdot e \quad \text { und } \quad p=\frac{d \cdot q}{l} \cdot e,
\end{gathered}
$$

wenn $e$ den Elastizitätsmodulus bezeichnet. Wir erhalten demnach: $P: p=Q: q$.

Das Verhältnis von $Q: q$ kann man aber annähernd gleich dem Verhältnis der Radien des Mittelquerschnittes des grossen und kleinen Ballons im aufgeblähten Zustande setzen, also

$$
P: p=R: r, \quad p=\frac{P \cdot r}{R} .
$$

Dieser Mittelquerschnitt des grossen Ballons $R^{2} \pi$ sei gleich $s^{2}$. Denkt man sich nun die Kreisfläche $R^{2} \pi$ erfüllt von einer grossen

1) Der Querschnitt der Wandungen ist in der Mitte am grössten und nimmt nach den Enden zu ab. Zur Vereinfachung ist daher ein mittlerer Querschnitt angenommen. 
Zahl $n$ sich berührender sehr kleiner Kreise vom Radius $r$, so wird diese Zahl annähernd auch gleich der Zahl Kreise sein, welche das Quadrat $s^{2}$ erfüllen, also:

$$
s=2 r \sqrt{n}, \quad s^{2}=4 r^{2} n \text { und } R^{2} \pi=4 r^{2} n .
$$

Statt des grossen Ballons mit dem Radius $R$ mögen wir nun im gefültten Zustande $v$ kleine Ballons mit dem Radius $r$ haben, die sich gegenseitig berühren.

Es ist also:

$$
r=\frac{R}{2} \sqrt{\frac{\pi}{n}}
$$

und demnach:

$$
p=P \cdot \frac{1}{2} \sqrt{\frac{\pi}{n}} .
$$

Haben wir also $n$ kleine Ballons, so wird die Gesamtkraft derselben:

$$
n \cdot p=P \cdot \frac{1}{2} \sqrt{n \cdot \pi}
$$

Die Kraft wächst also proportional mit der Quadratwurzel aus der Zahl der Ballons. Non hatten wir aus dem mit dem Kautschukballon angestellten Versuche gefunden, dass die Kraft $P$ ungefähr gleich dem innern Druck ist, beides auf die Flächeneinheit bezogen. Dies übertragen wir auch auf jeden der kleinen Ballons; $P$ nehmen wir als den berechneten osmotischen Druck gleich $6 \mathrm{~g}$ an und die Kraft einer Muskelzuckung wie früher zu $600 \mathrm{~g}$.

Es müsste also sein:

$$
6 \cdot \frac{1}{2} \sqrt{n \cdot \pi}=600 \text {. }
$$

Hieraus erhalten wir $n=12733$; d. h. also, es müssten auf $1 \mathrm{qcm}$ Muskel 12733 kleine ballonförmige Blasen von der angenommenen Beschaffenheit kommen, wenn durch den osmotischen Druck bei der Zuckung die Muskelkraft von $600 \mathrm{~g}$ erzengt werden sollte.

Ich hatte nun bei einer anderen Gelegenheit auf 1 qinm Querschnitt des Froschmuskels 139,6 Fasern gemessen, also auf 1 qcm 13960 Fasern $^{1}$ ). Es könnte also der Durchmesser eines Bläschens sogar den einer Muskelfaser annehmen, so würden wir schon die

1) Siehe: Die Energie des Muskels als Oberflächenenergie, dieses Archiv Bd. 85 S. 293. 1901. 
Muskelkraft erreicht haben. Dies würde also a fortioni der Fall sein, wenn wir den Bläschen nur den Durchmesser der Fibrillen zuerteilen würden.

III.

Das Resultat der vorangegangenen Betrachtungen und Berechnungen ist folgendes:

Der osmotische Druck, welcher in den kontraktilen Elementen der Muskelfaser bei der Kontraktion anwächst, würde ausreichen, die Muskelkraft zu erzeugen, wenn die hierzu notwendige Bedingung erfüllt wäre, welche darin bestände, dass diese Elemente aus kleinen mit längsgefalteten Wandungen versehenen Bläschen in bestimuter Anordnung gebildet wären.

Was hiernach rom osmotischen Druck gilt, das würde selbstverständlich auch von einem durch Quellung erzeugten Druck gelten, wenn wir dieselben mechanischen Bedingungen voraussetzten. Bekanntlich können durch Quellung sehr bedeutende Drucke erzeugt werden. Es kann also keinem Zweifel unterliegen, dass eine Zunahme des Quellungsdruckes in den angenommenen kontraktilen Elementen die Muskelkraft erreichen kann. Man müsste sich vorstellen, dass diese Elemente mit einer Kolloidlösung erfüllt sind, welche bei der Kontraktion eine chemische Veränderung erleidet und dabei einen höheren Quellungsdruck annimmt ${ }^{1}$ ).

Die unerlässliche Bedingung aber für das Zustandekommen der Kontraktion auch unter dieser Annahme besteht darin, dass die kontraktilen Elemente die oben angegebene Struktur und Anordnung besitzen. Bevor daher weitere Konsequenzen an eine solche Hypothese geknüpft werden, halte ich es für notwendig, die Frage mit histologischen Mitteln zu behandeln. Obgleich ich weiss, dass nach allen bisherigen bistologischen Untersuchungen eine solche Struktur, wie sie nach jener Annahme verlangt wurde, nicht bemerkt worden ist und mir dieselbe nach eigener Anschauung höchst unwahrscheinlich vorkommt, möcbte ich doch nicht versäumen, die Herren Histologen von Fach zu einer Entscheidung aufufordern.

1) Diese Vorstellung unterscheidet sich von der Engelmann'schen darin, pass letztere die Quellungskraft durch die vom chemischen Prozess herrührende Wärme wachsen lässt, wobei die quellbare Substanz sich selbst nicht chemisch zu verändern braucht, 
Sollte die Entscheidung so ausfallen, dass die mikroskopisch wahrnehmbaren kontraktilen Elemente der Muskelfasern unmöglich aus Bläschen mit longitudinal gefalteten Wandungen bestehen können, so würden damit die osmotische und Quellungstheorie der Kontraktion in dem behandelten Sinne zu verwerfen sein. Es würde sich damit die Wage zugunsten der Oberflächenspannungstheorie erheblich neigen, wie ich letztere schon früher behandelt habe. Diese Theorie ist von der Gestalt der kontraktilen Elemente nur insoweit abhängig, als bei der Verkürzung in ihrer Längsrichtung ihre Oberfläche kleiner werden muss. Fine Grenze für die Verkürzung wäre, wie es der Wirklichkeit entspricht, auch bei dieser Theorie in dem Punkte gegeben, in welchem die Oberfläche ein Minimum erreiclt hat, aber niemals könnte darüber hinaus eine Wiederverlängerung eintreten, wie es bei jenen beiden Theorien möglich wäre.

Bei der osmotischen und Quellungstheorie sind Volumenergien im Spiele. In solchem Falle macht es besondere Schwierigkeit zu erklären, dass das Volumen des arbeitenden Körpers sich nicht ändert. Sie erfordern ferner eine ausserordentlich schnelle Bewegung von Wassermolekülen durch Diffusion. Auch von diesen Schwierigkeiten ist die Oberflächenspannungstheorie frei. Dass aber andere Energieformen als die osmotische, Quellungsenergie oder Oberflächenenergie in dem Muskel aus der chemischen Energie entstehen, um die Muskelarbeit zu erzeugen, ist nach unseren heutigen Kenntnissen im höchsten Grade unwahrscheinlich. 